\title{
Reconstruction of the Medial Canthus Using an Ipsilateral Paramedian Forehead Flap
}

\author{
Jin Hyung Kim, Jeong Min Kim, Jang Wan Park, Jae Ha Hwang, Kwang Seog Kim, \\ Sam Yong Lee \\ Department of Plastic and Reconstructive Surgery, Chonnam National University Medical School, Gwangju, Korea
}

Background The medial canthus is an important area in determining the impression of a person's facial appearance. It is composed of various structures, including canthal tendons, lacrimal canaliculi, conjunctiva, the tarsal plate, and skin tissues. Due to its complexity, medial canthal defect reconstruction has been a challenging procedure to perform. The contralateral paramedian forehead flap is usually used for large defects; however, the bulkiness of the glabella and splitting at the distal end of the flap are factors that can reduce the rate of flap survival. We reconstructed medial canthal defects using ipsilateral paramedian forehead flaps, minimizing glabellar bulkiness.

Methods This study included 10 patients who underwent medial canthal reconstruction using ipsilateral paramedian forehead flaps between 2010 and 2012. To avoid an acute curve of the pedicle, which can cause venous congestion, we attempted to make the arc of the pedicle rounder. Additionally, the pedicle was skeletonized from the nasal root to the glabella to reduce the bulkiness.

Results All patients had basal cell carcinoma, and 3 of them had recurrent basal cell carcinoma. All of the flaps were successful without total or partial flap loss. Two patients developed venous congestion of the flap, which was healed using medicinal leeches. Four patients developed epiphora, and 2 patients developed telecanthus.

Conclusions Large defects of the medial canthus can be successfully reconstructed using ipsilateral paramedian forehead flaps. In addition, any accompanying venous congestion can be healed using medicinal leeches.

Keywords Forehead / Surgical flaps / Leeches / Carcinoma
Correspondence: Sam Yong Lee Department of Plastic and Reconstructive Surgery, Chonnam National University Medical School, 42 Jebong-ro, Dong-gu, Gwangju 501-757, Korea

Tel: +82-62-220-6363

Fax: +82-62-227-1639

E-mail: sylee@jnu.ac.kr
No potential conflict of interest relevant to this article was reported.

\section{INTRODUCTION}

The medial canthus comprises the medial periorbital area, and it is present at a fixed distance from the facial midline. It is supported by medial canthal tendons attached to the frontal process of the maxilla. The medial canthus is anatomically important because the lacrimal puncta, through which tears flow, are located above and below the medial canthus, and the lacrimal canaliculi, which are linked to the lacrimal sac, are distributed in the medial canthus. In addition, it is easy to note a congenital deformity if the intercanthal distance is longer or shorter than normal. When certain diseases cause defects in the medial canthal area, the medial canthus requires reconstruction with the surrounding tissue so that it will have a normal shape and function. The com- 
plications that must be overcome during the reconstruction of the medial canthus include cosmetic defects, which essentially accompany excess tear secretion, and mobile disability caused by abnormally thick skin [1].

If the defect caused by the excision of malignant skin cancer (a major cause of medial canthus skin defects) is large, the paramedian forehead flap is preferred [2]. Reconstruction using a paramedian forehead flap is performed by utilizing the contralateral supratrochlear artery as the pedicle because it has the merit of not being bent severely due to its large rotation arc. When this method is used, the nose dorsum can bulge because the pedicle crosses the upper nose. In order to prevent this, it is possible to cross above the skin as passing over a bridge [3]. However, a drawback of this method is that the area should be divided and adjusted after 2 to 3 weeks. Therefore, in this study, we applied a paramedian forehead flap method utilizing the ipsilateral supratrochlear artery for medial canthus defect reconstruction, instead of a common paramedian forehead flap method that utilizes the contralateral supratrochlear artery. We dissected the subcutaneous tissue around the pedicle as much as possible and had the flap pass through the side of the nose, instead of the nose dorsum, so that the bulging of the nose dorsum would be minimized. We treated venous congestion by using medicinal leeches and observed the results.

\section{METHODS}

\section{Study subjects}

The subjects of this study included 10 patients with medial canthus skin defects who were admitted to this department and underwent operations between January 2010 and December 2012. These 10 patients had basal cell carcinoma, and 3 of them had recurrent basal cell carcinoma. The flap method used for all of the patients was the ipsilateral paramedian forehead flap method.

\section{Technique}

All of the patients underwent the operation under general anesthesia. When excising the lesion, the safety margin was expanded by $\geq 5 \mathrm{~mm}$ and the absence of cancer cells at the margin was confirmed by frozen biopsy. If the cross-section of the lacrimal punctum and lacrimal canaliculus was exposed during the excision, the boundary tissue was sutured with the lacrimal canaliculus buried inward. If the lacrimal sac was cut, the mucous membrane and flap were separated by burying and suturing both ends of the lacrimal sac.

The location of the supratrochlear artery was identified by using Doppler ultrasound at the medial $1 / 3$ of the ipsilateral superior orbital rim. We tracked the path of the supratrochlear artery toward the forehead and marked the location where the last pulsation was heard. The distance from the superior orbital rim to the forehead was measured as well as the distance from the superior orbital rim to the defect, and a flap with the same size and shape as the defect was designed. A zigzag-shaped incision line was designed from the superior orbital rim to the flap. The distal area of the flap was incised down to the subcutaneous fat layer, and the supraperiosteal layer was dissected so that the subcutaneous tissue with relatively good vasculature would remain for skin grafting. After incising along the zigzag incision line from the flap toward the pedicle origin, we performed a subdermal dissection of both sides up to $10 \mathrm{~mm}$ above the superior orbital rim while protecting the supratrochlear artery. At a location 10 $\mathrm{mm}$ above the superior orbital rim, the dissection went under the periosteum so that the pedicle would remain between the subdermal plane and the infraperiosteal plane. Then the flap and pedicle were turned upside down, and the dissection proceeded carefully with a loupe so that the running of the supratrochlear artery could be identified, and the subcutaneous tissue around the pedicle was excised as much as possible.

After elevating and setting the flap at the defect, the area where the zigzag sutured incision line was located above the pedicle was manipulated so that the pedicle would not receive pressure when the incised skin was sutured at a later time. To prevent hematoma, drainage tubes were inserted. If the patient had many wrinkles, a wider range of the forehead donor site was dissected and the elongated skin was pulled for primary closure (Fig. 1). If closure was not possible, a full-thickness skin graft was performed from the postauricular area (Fig. 2). At the recipient site, congestion by hematoma was prevented by inserting drainage tubes into the flap from 4 directions.

\section{RESULTS}

The patients were aged 49 to 83 years, and the mean age was 71 years. There were 6 men and 4 women. The area of the defect was the right medial canthus in 6 patients and the left medial canthus in 4 patients. The mean interval from diagnosis of the disease to the operation was 18.5 months. All of the patients had basal cell carcinoma confirmed by biopsy ( 3 patients had recurrent basal cell carcinoma).

All of the patients underwent the operation under general anesthesia, and the mean hospital stay was 12 days. All of the flaps survived, and there were no areas of total or partial necrosis. Two patients who showed venous congestion were treated using medicinal leeches. Four patients had epiphora, and 2 patients showed telecanthus $\geq 3 \mathrm{~mm}$ farther from the midline than the normal side (Table 1). 

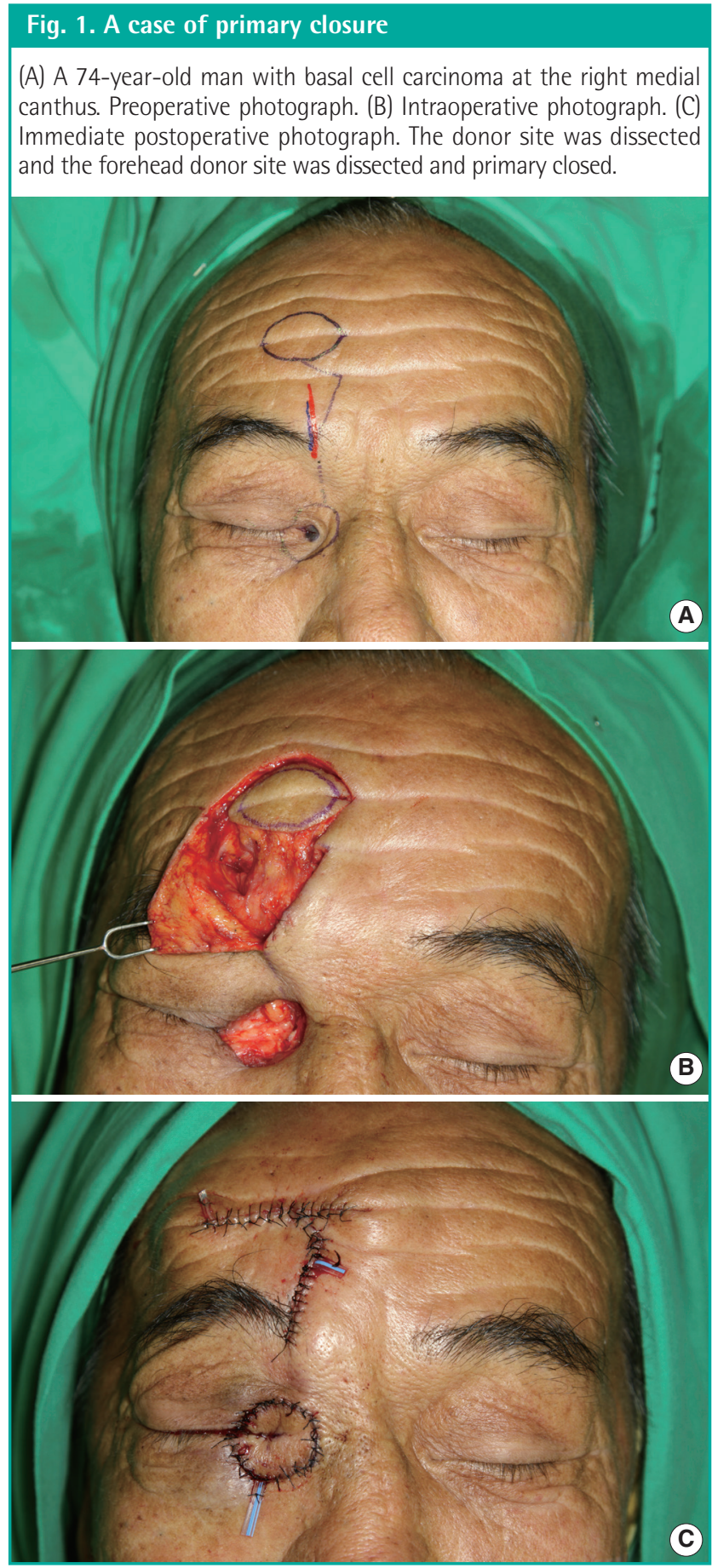

The mean size of the defect at the donor site was $5.6 \mathrm{~cm}^{2}$. Four patients had primary closure by dissecting the surrounding tissue, and 6 patients received full-thickness skin grafts from the postauricular area. All of the patients recovered without any severe complications such as wound dehiscence or flap necrosis.

\section{DISCUSSION}

The medial canthus, or the space between the eyes, determines

\section{Fig. 2. A case of full thickness skin graft}

(A) A 71-year-old man with basal cell carcinoma at the right medial canthus. Preoperative photograph. (B) Intraoperative photograph. (C) Immediate postoperative photograph. A full thickness skin graft was performed from the postauricular area.
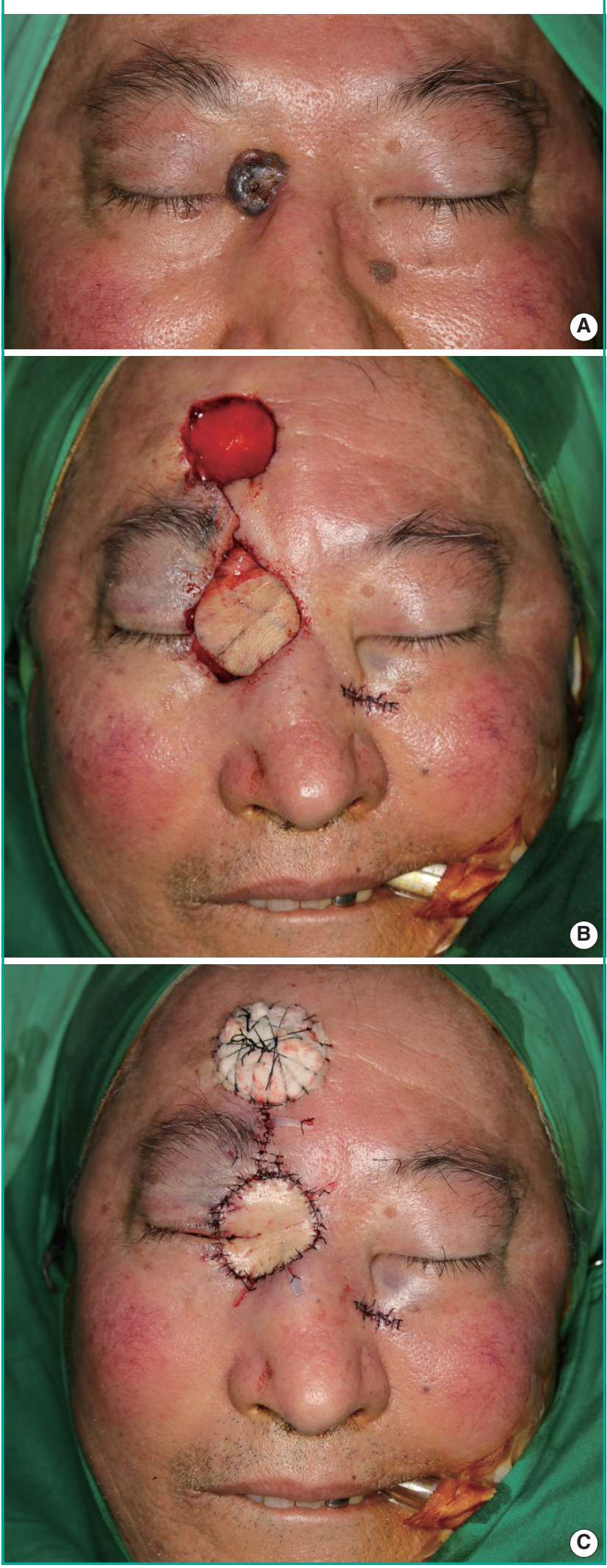
Table 1. Patient characteristics $(n=10)$

\begin{tabular}{|c|c|c|c|c|c|}
\hline Case & Age $(y r) / S e x$ & Cancer type & Defect size $\left(\mathrm{cm}^{2}\right)$ & Donor healing & Complications \\
\hline 1 & $72 / F$ & $\mathrm{BCC}$ & 5.4 & FTSG & - \\
\hline 2 & $78 / F$ & Recurred BCC & 4.0 & Primary closure & - \\
\hline 3 & $49 / M$ & $\mathrm{BCC}$ & 6.3 & FTSG & Epiphora, telecanthus ${ }^{\text {a) }}$ \\
\hline 4 & $70 / F$ & $\mathrm{BCC}$ & 4.0 & Primary closure & Venous congestion \\
\hline 5 & $83 / F$ & $\mathrm{BCC}$ & 5.7 & FTSG & Epiphora \\
\hline 6 & $74 / \mathrm{M}$ & Recurred BCC & 1.7 & Primary closure & - \\
\hline 7 & $74 / M$ & $\mathrm{BCC}$ & 3.0 & Primary closure & Bone exposure ${ }^{b)}$ \\
\hline 8 & $71 / M$ & Recurred BCC & 4.0 & FTSG & Venous congestion \\
\hline 9 & $68 / M$ & $\mathrm{BCC}$ & 5.9 & FTSG & Epiphora \\
\hline 10 & $71 / M$ & BCC & 16.0 & FTSG & Epiphora, telecanthus \\
\hline
\end{tabular}

the first impression and even the character of an individual because it is the first site that we gaze upon when we face another person. The distance of the vertical line running from the exact center of the nose to the medial canthus should ideally be identical on both sides, and the medial canthi should be symmetrical. Excessive distance could be caused by a congenital abnormality or past trauma.

As society ages and the number of aged individuals increases, the number of patients with various cancers is also increasing. Furthermore, there is an increasing trend in the number of patients with skin cancer caused by ultraviolet radiation, including exposure to sunlight. Among the skin cancers occurring on the face of elders, basal cell carcinoma is the most common. In addition, $86 \%$ of basal cell carcinomas occur on the face and $14 \%$ occur around the eyes [4].

When basal cell carcinoma occurs on the medial canthus, which is not a 2-dimensional structure like the forehead or cheek, the tumor and tissue around the lesion should be removed until there are no more cancer cells or tumor tissue. Furthermore, the surrounding structure might need reconstruction. At this time, tissues including the lacrimal punctum, lacrimal canaliculus, medial canthal ligament, lacrimal sac, caruncle, and a portion of the conjunctiva, may get damaged [5]. Epiphora caused by damage in the lacrimal punctum, lacrimal canaliculus, and lacrimal sac is an unavoidable complication. Injury to the lacrimal canaliculus leads to scarring and stenosis, which in turn can cause complete or incomplete obstruction of the canaliculus. A medial canthal tendon injury can also cause epiphora by disturbing the normal posteromedial pull of the lid. To minimize the injury to the lacrimal system, tumor resection was performed with a minimum safety margin around the eyelid, and the margin was confirmed by frozen biopsy. When a lacrimal canaliculus injury was suspected or its partial injury was identified, a prophylactic nasolacrimal intubation was performed using a Mini-monoka. When a direct defect to the lacrimal system was discovered, the boundary tissue was sutured with the lacrimal canaliculus buried inward and a secondary reconstruction of the nasolacrimal system was planned after a follow-up period, however, no patient complained of epiphora to the extent that it interfered with daily life.

If the distance from the midline of the face to the normal medial canthus and the distance from the midline of the face to the reconstructed medial canthus differ, the patient may experience telecanthus along with serious cosmetic issues and changes in the visual field. Therefore, the actual distance should be measured, and the flap should be designed carefully during the reconstruction operation. Even in cases where a direct injury to the medial canthal tendon was discovered, instead of immediate repair of the canthal tendon, an epicanthoplasty during the follow-up period was planned, as immediate repair surgery could affect flap survival.

In medial canthal reconstruction, various techniques using a local flap such as rhomboid flap, subcutaneous pedicle V-Y advancement flap, upper eyelid myocutaneous flap from the ipsilateral or contralateral side, V-Y glabellar flap, forehead flap, and combinations of these flaps have been reported [2]. These procedures have advantages and disadvantages and are selected by surgeons according to the defect size, location, and donor site morbidity. Since the majority of the cases involve periosteum or bone exposure, difficulties with skin graft survival were expected, and even if the skin graft took well, a significant depression was expected with a poor cosmetic outcome. In addition, most cases had partial upper and lower eyelid defects and skin grafting after an eyelid defect would limit opening and closing eye motion. Taking into consideration these drawbacks of the skin graft, the flap surgery was the preferred procedure. V-Y-S plastic surgery was performed when the skin defect around the medial canthus was small. This had the advantage of a zigzag scar; however, this procedure cannot be applied when the defect is large [6]. The V-Y glabella flap method can be applied when 
the lesion diameter is $\leq 15 \mathrm{~mm}$. Because this method uses the wrinkles of the glabella, the donor site scar after reconstruction remains in linear form. Therefore, the V-Y glabella flap is recommended as a good method. If the lesion size is large, it is possible to reconstruct the defect with the paramedian forehead flap method.

The paramedian forehead flap method elevates the flap with the supratrochlear vessels and nerves. The supratrochlear artery rises from the supraorbital foramen toward the forehead under the periosteum. After it runs to the eyebrow above the periosteum, it receives randomized vascularity at the infradermal layer. It is possible to design a flap in the desired shape from the center of the forehead after applying a linear incision line from the superior orbital rim to the forehead.

A common method involves using the contralateral supratrochlear artery across the exact center of the nose to minimize torsion and obstruction of the pedicle by an acute rotation arc. The drawback of this method is that the area between both sides of the medial canthus can bulge because of the pedicle running across the center of the nose. The pedicle crossing the skin of the nose like a tube requires other operations, including flap division, after 3 weeks.

In order to overcome this drawback, a method was devised in which the paramedian forehead flap is elevated at the ipsilateral forehead, the supratrochlear artery and vein are skeletonized by removing as much unnecessary subcutaneous tissue around the pedicle as possible, and the defect is covered by passing through a tunnel under the skin. In many cases, the arteries of the face do not clearly accompany the vena comitans. The path of the supratrochlear artery can be confirmed using Doppler ultrasound; however, since the vein runs together with the artery in a separated state instead of as a clear vena comitans, it is difficult to completely skeletonize the artery and vein.

If a 10-mm width of the surrounding subcutaneous tissue is elevated, focusing on the artery path, the venous circulation will be included in it. To remove the factors that compress the pedicle, a portion of subcutaneous tissue inside the subcutaneous tunnel, which is on the path to the medial canthus defect, was excised. The flap will not have venous congestion when the flap is naturally located on the defect; therefore, we provided sufficient distance so that the flap would not receive tension. The medial canthus is closely attached to the medial canthal ligament, and it has a slightly pressed shape at the side of the nose. However, the forehead flap is thick; therefore, if the forehead flap would be simply sutured to the defect in the medial canthus, it would be thicker than the outside eyelid, and the mobility of the upper and lower eyelids would be impaired. As a solution to this, the distal part of the flap to be located at the medial canthus was made thin by removing the subcutaneous fat, and the bottom of it was fixed at the medial canthal tendon. In preparation for the possibility of the lid fissure becoming narrower due to scarring at a later time, the incision was created 2 $\mathrm{mm}$ longer than the lid fissure incision.

Further, in order to prevent a union caused by scarring, the upper and lower eyelid conjunctiva were sutured to each split supraorbital and infraorbital rim of the flap. The suture line of the upper and lower eyelid and flap was oblique instead of vertical to prevent ectropion due to a vertical line scar. The patients experienced no serious inconvenience in closing their eyes when the ratio of the flap to upper eyelid was 1:4; however, if it was greater than that, the patients experienced epiphora because the lid fissure was always in an open state.

When the flap was rotated while the pathway of the supratrochlear artery and vein was not clear, congestion was com-

(A) Thirty-six-month postoperative photograph with eyes open. (B) Postoperative photograph with eyes closed. Severe flap bulkiness is not present.
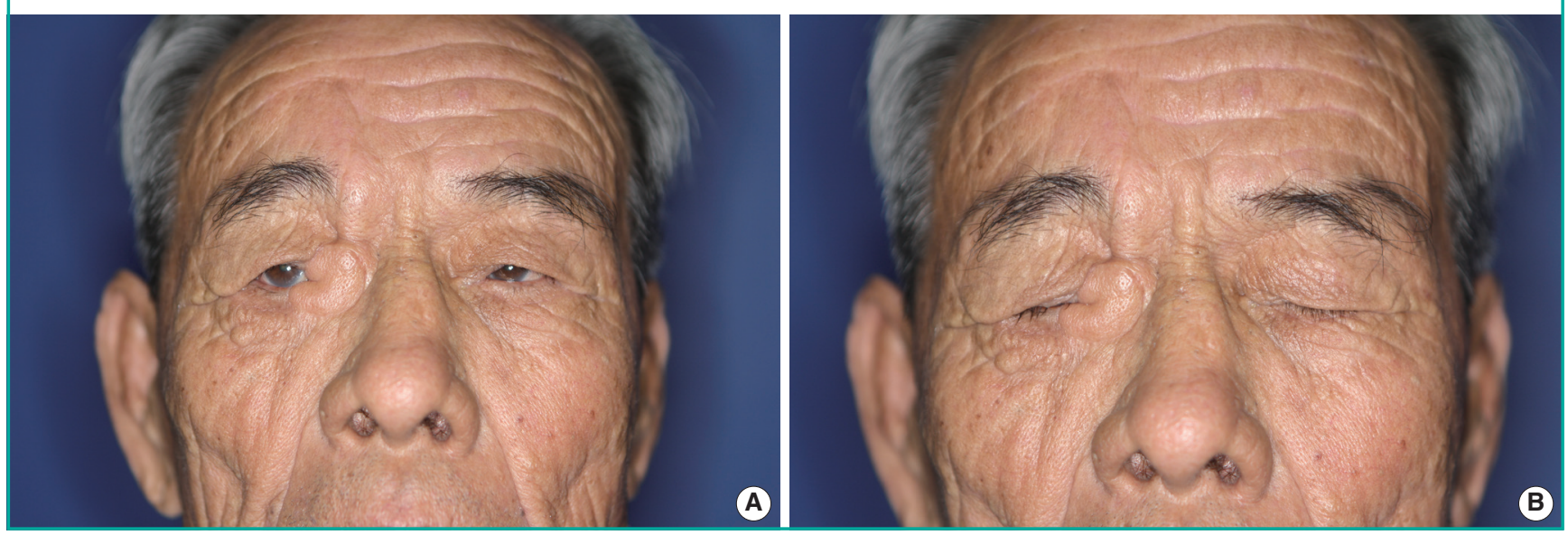
mon; however, congestion could be alleviated by applying medicinal leeches. When venous congestion occurred, stitches of a part of the thread suturing the flap were first removed to reduce the tension on the flap, and the congested flap was checked after 1 hour. In the 2 cases in which congestion occurred, no improvement was found in the post-intervention observation, and leech application was necessary. To address venous congestion, Akan et al. [7] proposed a method for inducing continuous bleeding of the donor site under intravenous injection of heparin after harvesting a split thickness skin graft from a distal portion of the flap. A similar method involves laceration with a blade to induce continuous bleeding. Medicinal leech application is quite a good therapeutic approach when the congestion is temporary and the flap is not large [8]. In the case of a forehead flap, given the small flap size and low amount of bleeding required to relieve congestion, leech application is a very effective method.

Because of the position of the flap close to the eye, care was taken to prevent the leech from migrating from the flap toward the eyeball, which could result in injury to the eyeball. To prevent the leech from approaching the eyeball, tapes were attached to the upper and lower eyelids, which were kept closed, and the eyelids were then covered with gauze. The leech application itself lasted from 15 to 30 minutes, and continuous bleeding after leech application was ensured by covering the bleeding point with heparin-soaked gauze.

Nasal dorsum bulging caused by the pedicle crossing the nasal dorsum was not obvious during the early period of the operation, and the nose dorsum profile was able to retain its original shape. There was sensory loss at the forehead when a paramedian forehead flap method was used ipsilaterally or contralaterally; however, we sought the understanding of the patient by explaining the likelihood of this in advance.

We left subcutaneous tissue, including the periosteum, at the donor site defect and applied the full-thickness skin graft above it. When the flap is elevated, leaving much tissue at the forehead has the advantage of reducing flap thickness and donor site depression, but is likely to impede circulation in the flap. Therefore, the first priority was given to flap survival, and only a part of the tissue above the periosteum was left. Follow-up observations confirmed considerable improvement in flap bulkiness (Fig. 3), and the forehead depression that occurred in some cases was acceptable to the patients because it had been explained to them preoperatively.

In a few patients, necrosis or exposure of the bone occurred. One patient had bone exposure and skin loss at the donor site after a full-thickness skin graft. We removed the necrotic skin and dressed the area with Aquacel Ag (Convatec, Skillman, NJ, USA). After 4 weeks, we covered the bone-exposed area with epithelial cells regenerated from the surrounding normal skin.

\section{REFERENCES}

1. Bostwick J 3rd, Vasconez LO, Jurkiewicz MJ. Basal cell carcinoma of the medial canthal area. Plast Reconstr Surg 1975; 55:667-76.

2. Onishi K, Maruyama Y, Okada E, et al. Medial canthal reconstruction with glabellar combined Rintala flaps. Plast Reconstr Surg 2007;119:537-41.

3. Guzel MZ. The turnover subdermal-periosteal median forehead flap. Plast Reconstr Surg 2003;111:347-50.

4. Shanoff LB, Spira M, Hardy SB. Basal cell carcinoma: a statistical approach to rational management. Plast Reconstr Surg 1967;39:619-24.

5. Mustarde JC. Surgery of the medial canthus. In: Mustarde JC, editor. Repair and reconstruction in the orbital region. 3rd ed. Edinburgh: Churchill Livingstone; 1991. p.255-81.

6. Argamaso RV. V-Y-S-plasty for closure of a round defect. Plast Reconstr Surg 1974;53:99-101.

7. Akan IM, Yildirim S, Gideroglu K. Salvage of flaps with venous congestion. Ann Plast Surg 2001;46:456.

8. Conforti ML, Connor NP, Heisey DM, et al. Evaluation of performance characteristics of the medicinal leech (Hirudo medicinalis) for the treatment of venous congestion. Plast Reconstr Surg 2002;109:228-35. 